\title{
Una propuesta de aprendizaje basada en el periodismo- acción en el aula
}

\section{A learning proposal based on action journalism in classroom}

Francisco BaEna SÁnCHEZ

ORCID: https://orcid.org/0000-0002-5304-7924

Universidad de Sevilla

Departamento de Periodismo 1

frbaena@us.es

Fecha de recepción: 17-11-2019.

Fecha de aceptación: 23-11-2019.

DOI: http://dx.doi.org/10.12795/9788447221912.074

Pp.: $1705-1730$ 


\section{Resumen}

Este trabajo describe el diseño, la experimentación y la evaluación del Ciclo de Mejora en el Aula (CIMA) que he aplicado en la asignatura Historia del Periodismo Universal en la Universidad de Sevilla. Propongo un modelo metodológico basado en el aprendizaje colaborativo y en el periodismo-acción en el aula. Mediante la simulación del trabajo en una redacción periodística, pretendo crear las condiciones adecuadas para que los estudiantes aprendan a pensar y actuar como un periodista. He evaluado el aprendizaje mediante un cuestionario de elección múltiple y mediante una rúbrica para calificar la práctica del reportaje. Esto me ha permitido comparar sus conocimientos iniciales y finales, así como representar gráficamente esta evolución mediante escaleras. Los principios didácticos que han guiado la experimentación se corresponden con un modelo alternativo centrado en el alumno y el aprendizaje.

Palabras claves: Historia del Periodismo Universal, Grado en Periodismo, docencia universitaria, experimentación docente universitaria.

\section{Abstract}

This paper describes an Improvement Cycle in Classroom (ICIC) that I designed and experimented on World Journalism History subject at the University of Seville. My teaching methodology is based on collaborative learning and action journalism in classroom. By simulating work in a newsroom, I expect students to learn to think and behave as a journalist. I assessed students learning through a multiple-choice question paper and through the article practical rubric. These tools allowed me to compare their initial and final knowledge and to show graphically their progress by learning stairs. Didactic principles that gave ICIC guidance agree with an alternative methodology focused on students and learning.

Keywords: World Journalism History, Journalism Degree, Higher Education Teaching, Innovation in Higher Education Teaching.

Jornadas de Formación e Innovación Docente del Profesorado | № 2 (2019) Esta obra se distribuye con la licencia Creative Commons Reconocimiento-NoComercial-SinObraDerivada 


\section{Contexto de aprendizaje}

La asignatura en la que he implementado el Ciclo de Mejora en el Aula (en adelante, CIMA) se llama «Historia del Periodismo Universal» y se inscribe en el segundo curso del Grado en Periodismo que se imparte en la Facultad de Comunicación de la Universidad de Sevilla. Se trata de una materia obligatoria y consta de 6 créditos ECTS que se traducen en 60 horas presenciales de clases teórico-prácticas (a razón de cuatro horas por semana divididas en dos días). Además de coordinador, soy el profesor responsable de tres grupos de docencia, de los cuatro que tiene la asignatura. Experimenté el ciclo, de 16 horas de duración, entre el 8 y el 30 de octubre de 2019.

El número de estudiantes que han participado en el CIMA ha sido de 199: 82 en el grupo 1, 68 en el grupo 3 y 49 en el grupo 4. Más allá de estos datos cuantitativos, me gusta comenzar la asignatura con los estudiantes. Por ese motivo, el primer día de clase, utilizo la aplicación Kahoot para realizar varias encuestas preliminares, de carácter anónimo, que me permiten tener una primera impresión personal del grupo (edad, sexo, lugar de origen, situación laboral), así como conocer mejor sus experiencias previas de aprendizaje, sus intereses profesionales, sus expectativas acerca de la asignatura o sus hábitos y prejuicios en relación con los medios de comunicación y el periodismo.

El aula destinada a las clases teóricas, que se imparten el primer día de la semana (martes), tiene una capacidad para 120 personas y es un espacio de aprendizaje absolutamente decimonónico, propicio para desarrollar un modelo metodológico basado en la transmisión directa del contenido a los estudiantes. El aula destinada a las clases prácticas, a las que dedicamos el segundo día de la semana (miércoles), es un aula de informática con unos 35 ordenadores disponibles y capacidad para 70-80 personas. 
Al igual que el aula de teoría, constituye un espacio de aprendizaje poco idóneo para la innovación docente.

El ciclo anterior, de 8 horas de duración, lo implementé en la misma asignatura durante el mes de octubre de 2017. Los principios didácticos que entonces guiaron aquella experimentación fueron dos: el primero, crear un entorno para el aprendizaje crítico natural; y, el segundo, atraer a los estudiantes al razonamiento disciplinar, para lo que se propuso el modelo metodológico de investigación-acción en el aula.

Una de las novedades que he introducido en el CIMA actual ha consistido en revisar el modelo metodológico, en el sentido de que el aula ya no simule tanto el trabajo en un laboratorio como en una redacción periodística, de manera que se promueva el aprendizaje de competencias profesionales y no exclusivamente académicas. A partir de la experiencia obtenida en el ciclo anterior, he seguido definiendo mi modelo didáctico personal y transformando mi práctica docente, en el sentido de facilitar procesos más participativos, creativos y útiles en la formación académica y profesional del alumnado.

\section{Diseño previo del CIMA}

\section{Mapa de contenidos y problemas}

La unidad didáctica que se aborda en el CIMA se titula «El periodismo en la Edad Moderna: de los orígenes a la conquista de la libertad de prensa (siglos XVI-XVIII)》. El problema principal, que los estudiantes deben resolver, es de tipo académico y se formula así: «las bases históricas del periodismo actual aparecen de manera muy clara en el contexto histórico de la Edad Moderna». Para 
estimular el interés y la curiosidad de los estudiantes en torno a este problema, utilizo un juego de palabras basado en el célebre lema que empezó a popularizarse en esta época, como expresión ritual, cada vez que fallecía un monarca y le sucedía su legítimo heredero: «El periodismo ha muerto... ¡Larga vida al periodismo!». Además, el problema se plantea en el contexto disciplinar de la historia del periodismo, puesto que los expertos no se ponen de acuerdo sobre en qué momento histórico debe situarse su origen o nacimiento.

A continuación, puede visualizarse el mapa de contenidos y problemas que ha guiado el aprendizaje autónomo y colaborativo de los estudiantes en el aula. La trama destaca los principales núcleos de conocimiento, planteados en forma de preguntas, y muestra las interacciones más potentes y significativas entre estos problemas (PR) y los contenidos, que hemos diferenciado visualmente mediante colores: el morado, para los contenidos conceptuales (CC); el azul, para los contenidos procedimentales (CP); y el verde, para los contenidos actitudinales (CA). Los conceptos enmarcados sobre fondo naranja contienen preguntas de carácter secundario, que no hemos podido explicitar por una cuestión de espacio.

Jornadas de Formación e Innovación Docente del Profesorado | № 2 (2019) Esta obra se distribuye con la licencia Creative Commons 
FRANCISCO BAENA SÁNCHEZ

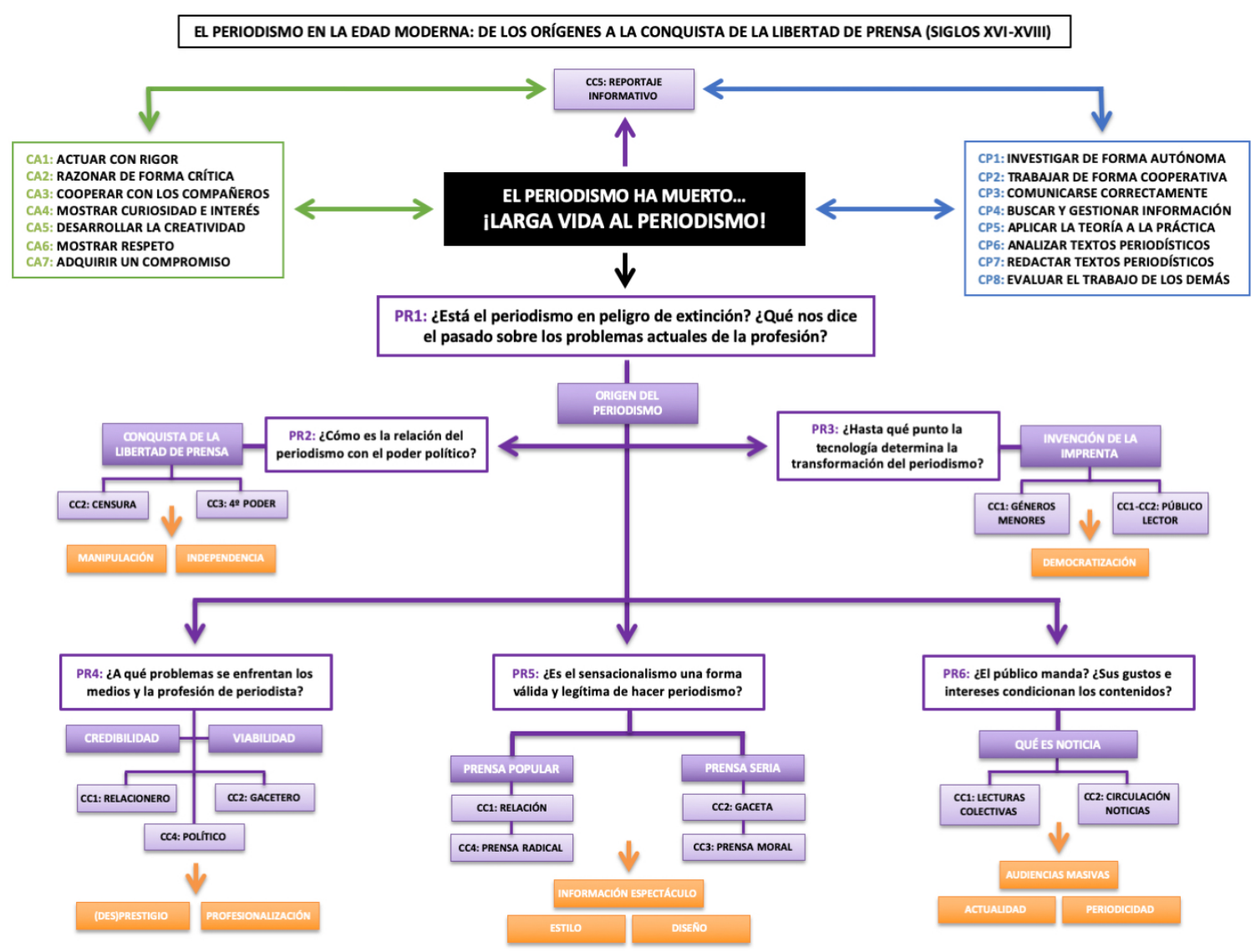

Figura 1. Mapa de contenidos y problemas diseñado para implementar el CIMA.

Jornadas de Formación e Innovación Docente del Profesorado I № 2 (2019)

(C) Esta obra se distribuye con la licencia Creative Commons

(c) $(7)($ EY Reconocimiento-NoComercial-SinObraDerivada

Internacional (CC BY-NC-ND 4.0.) 


\section{Modelo metodológico posible y secuencia de actividades programadas}

Hay dos premisas que subyacen al modelo metodológico que he diseñado para desarrollar el CIMA. Según la primera, el periodismo es historia, porque sin conocer su pasado no se pueden entender los problemas actuales de la profesión. De acuerdo con la segunda, la historia es noticia, puesto que la información de naturaleza histórica ocupa y preocupa tanto a los medios de comunicación como a la opinión pública. A partir de estas dos premisas, he adaptado el modelo metodológico de investigación-acción en el aula (Piqué y Forés, 2012; García Pérez, 2000). Mediante la simulación del trabajo en una redacción periodística, pretendo crear las condiciones adecuadas para que los estudiantes aprendan a pensar, analizar, redactar y concluir a la manera de un periodista. En este caso, por tanto, sería más apropiado hablar de periodismo-acción en el aula, pues considero a los estudiantes como un equipo de periodistas potenciales, en el que la colaboración hace posible un éxito mayor en su aprendizaje.

Las clases teóricas (TEO), a las que destino ocho horas, están pensadas para provocar la reacción de los estudiantes mediante la formulación de problemas y estudios de casos que les inviten a debatir y reflexionar. Sin embargo, es en las ocho horas de las clases prácticas (PRA) cuando cedo el control a los estudiantes, para que estos asuman un papel activo en su proceso de aprendizaje y desarrollen la propuesta de periodismo-acción en el aula.

En estas sesiones, aspiro a que los estudiantes se sientan periodistas por unas horas, de manera que se transformen algunos procesos profesionales, tales como la documentación, el análisis, el diseño, la redacción o la edición, en actividades de aprendizaje colaborativo y desempeñen roles que coinciden con los contenidos procedimentales diseñados. Estas tareas, completadas 
necesariamente fuera de clase, se han diseñado para que desarrollen habilidades profesionales, analíticas y creativas basadas en el trabajo autónomo.

Durante las cuatro semanas que dura el ciclo, los estudiantes deben elaborar-agrupados en equipos de 8-10 integrantes- un reportaje informativo de interés histórico sobre el origen del periodismo, en el que deben abordar los problemas del mapa. Además de recibir mi orientación en las clases prácticas, disponen de una guía en la plataforma de enseñanza virtual.

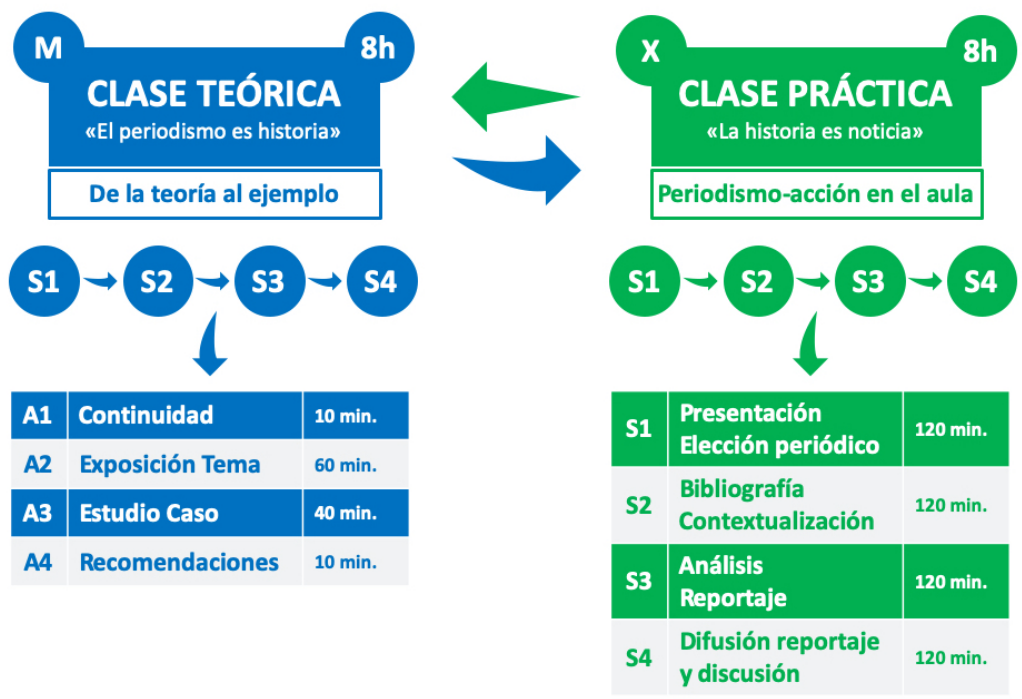

Figura 2. Modelo metodológico formalizado para el CIMA.

A continuación, se muestra, en orden cronológico, la secuencia completa de actividades que he diseñado para implementar el CIMA. Con estas actividades (A), pretendo aplicar coherentemente el modelo metodológico propuesto, de manera que los estudiantes trabajen los problemas y los contenidos formulados en el mapa.

Jornadas de Formación e Innovación Docente del Profesorado | № 2 (2019) Esta obra se distribuye con la licencia Creative Commons Reconocimiento-NoComercial-SinObraDerivada 
Tabla 1. Secuencia completa de actividades diseñadas para implementar el CIMA.

\begin{tabular}{|c|c|c|}
\hline Sesiones & Actividades formativas & Duración \\
\hline $\begin{array}{l}\text { Una semana } \\
\text { antes }\end{array}$ & $\begin{array}{l}\text { A1: Cuestionario inicial de preguntas cortas } \\
\text { PR1-PR6 / CC1-CC4 / CP1, CP4 / CA2, CA4, CA7 }\end{array}$ & 10 minutos \\
\hline TEO-1 & $\begin{array}{l}\text { A2: El nacimiento de la prensa en los } \\
\text { primeros tiempos de la imprenta } \\
\text { PR1, PR3-PR6 / CC1 / CP5, CP6 / CA2, CA4, CA7 }\end{array}$ & $\begin{array}{l}120 \\
\text { minutos }\end{array}$ \\
\hline \multirow{4}{*}{ PRA-1 } & $\begin{array}{l}\text { A3: Autoevaluación } \\
\text { CC5 / CP8 / CA7 }\end{array}$ & 10 minutos \\
\hline & $\begin{array}{l}\text { A4: Introducción al género periodístico del } \\
\text { reportaje } \\
\text { CC5 / CP7 / CA1, CA5 }\end{array}$ & $\begin{array}{l}30 \\
\text { minutos }\end{array}$ \\
\hline & $\begin{array}{l}\text { A5: Presentación de la práctica } \\
\text { CC1 / CP5, CP6 / CA2, CA4, CA7 }\end{array}$ & $\begin{array}{l}20 \\
\text { minutos }\end{array}$ \\
\hline & $\begin{array}{l}\text { A6: Elección de un periódico } \\
\text { CC1 / CP1, CP2, CP4 / CA1, CA3 }\end{array}$ & $\begin{array}{l}60 \\
\text { minutos }\end{array}$ \\
\hline TEO-2 & $\begin{array}{l}\text { A7: Fijación y evolución del gaceterismo en } \\
\text { el Estado absolutista moderno } \\
\text { PR1, PR2, PR4, PR6 / CC2 / CP5, CP6 / CA2, } \\
\text { CA4, CA7 }\end{array}$ & $\begin{array}{l}120 \\
\text { minutos }\end{array}$ \\
\hline \multirow[b]{2}{*}{ PRA-2 } & $\begin{array}{l}\text { A8: Consulta de la bibliografía recomendada } \\
\text { PR1-PR6 / CC1, CC2 / CP1, CP2, CP4 / CA1-CA3, } \\
\text { CA7 }\end{array}$ & $\begin{array}{l}60 \\
\text { minutos }\end{array}$ \\
\hline & $\begin{array}{l}\text { A9: Contextualización histórica de las } \\
\text { noticias } \\
\text { PR4-PR6 / CC1, CC2 / CP1, CP2, CP4 / CA1, CA3, } \\
\text { CA4 }\end{array}$ & $\begin{array}{l}60 \\
\text { minutos }\end{array}$ \\
\hline TEO-3 & $\begin{array}{l}\text { A10: Las batallas del periodismo británico } \\
\text { PR1, PR2, PR4, PR6 / CC3 / CP5, CP6 / CA2, } \\
\text { CA4, CA7 }\end{array}$ & $\begin{array}{l}120 \\
\text { minutos }\end{array}$ \\
\hline \multirow[b]{2}{*}{ PRA-3 } & $\begin{array}{l}\text { A11: Análisis del periódico } \\
\text { PR4-PR6 / CC1 / CP2, CP5, CP6 / CA2, CA3 }\end{array}$ & $\begin{array}{l}60 \\
\text { minutos }\end{array}$ \\
\hline & $\begin{array}{l}\text { A12: Elaboración de un reportaje de interés } \\
\text { histórico } \\
\text { PR1-PR6 / CC1-CC2 / CP2, CP3, CP7 / CA1-CA3, } \\
\text { CA5 }\end{array}$ & $\begin{array}{l}60 \\
\text { minutos }\end{array}$ \\
\hline
\end{tabular}

Jornadas de Formación e Innovación Docente del Profesorado | № 2 (2019) Esta obra se distribuye con la licencia Creative Commons Reconocimiento-NoComercial-SinObraDerivada Internacional (CC BY-NC-ND 4.0.) 


\begin{tabular}{|l|l|l|}
\hline \multirow{2}{*}{ TEO-4 } & $\begin{array}{l}\text { A13: La prensa política al servicio de la } \\
\text { revolución } \\
\text { PR2, PR4-PR6 / CC4 / CP5, CP6 / CA2, CA4, CA7 }\end{array}$ & $\begin{array}{l}120 \\
\text { minutos }\end{array}$ \\
\hline \multirow{5}{*}{ PRA-4 } & $\begin{array}{l}\text { A14: Difusión del reportaje y discusión de los } \\
\text { resultados } \\
\text { PR1-PR6 / CC1-CC2 / CP2, CP3, CP8 / CA6, CA7 }\end{array}$ & $\begin{array}{l}100 \\
\text { minutos }\end{array}$ \\
\cline { 2 - 4 } & $\begin{array}{l}\text { A15: Autoevaluación } \\
\text { CC5 / CP8 / CA7 }\end{array}$ & 10 minutos \\
\cline { 2 - 4 } & $\begin{array}{l}\text { A16: Cuestionario final de preguntas cortas } \\
\text { PR1-PR6 / CC1-CC4 / CP1, CP4 / CA2, CA4, CA7 }\end{array}$ & 10 minutos \\
\hline 48 horas & $\begin{array}{l}\text { A17: Entrega del reportaje } \\
\text { CP2 / CA3, CA6, CA7 }\end{array}$ & - \\
\hline
\end{tabular}

\section{Seguimiento del aprendizaje de los estudiantes}

He diseñado varios instrumentos de evaluación para seguir cómo evolucionan los modelos mentales de los estudiantes, es decir, para medir la progresión de su aprendizaje a lo largo del ciclo. El análisis de los resultados proporcionados por estos instrumentos no sólo me permitirá representar gráficamente esta evolución, sino también conocer en qué grado mi diseño ha promovido los aprendizajes previstos al principio y si los estudiantes han superado o no los obstáculos detectados.

El primer instrumento consiste en un cuestionario individual que se pasa al inicio y al final del ciclo y que está basado en los problemas y contenidos conceptuales del mapa. La prueba, que se responde en el aula a través de la plataforma de enseñanza virtual, consta de 10 preguntas cerradas de elección múltiple sobre: 1) la invención de la imprenta; 2) el control de la información; 3) la conquista de la libertad de prensa; 4) el periodismo como cuarto poder; 5) el relacionero y el gacetero; 6) el periodista político; 7) las relaciones y las gacetas; 8) la prensa política; 9) la difusión de las noticias; y 10) los públicos de la prensa. Cada pregunta tiene tres posibles respuestas, de las que al menos una es correcta. Las preguntas contestadas 
incorrectamente no restan puntos. En el cuestionario inicial, los estudiantes tienen la opción de seleccionar la respuesta «NS/NC» (no sabe, no contesta), para evitar que respondan al azar y así obtener información fiable sobre lo que realmente saben y piensan. El tiempo máximo para realizar la prueba es de 10 minutos. La calificación final de la prueba es sobre 10, por lo que cada pregunta vale un punto.

La evaluación del reportaje periodístico que los estudiantes realizan en las clases prácticas es el segundo instrumento diseñado para conocer y analizar la progresión de su aprendizaje. A continuación, se muestra la rúbrica utilizada para calificar su trabajo autónomo y colaborativo, según el modelo de periodismo-acción en el aula.

Tabla 2. Rúbrica para calificar las prácticas de la asignatura.

\begin{tabular}{|c|c|c|c|c|c|}
\hline \multirow{2}{*}{ CRITERIOS } & \multicolumn{5}{|c|}{ NIVELES DE RENDIMIENTO } \\
\hline & Muy mal & Mal & Regular & Bien & Excelente \\
\hline \multirow[b]{2}{*}{$\begin{array}{l}\text { Busca y } \\
\text { selecciona } \\
\text { información } \\
\mathrm{CP} 1, \mathrm{CP} 2, \mathrm{CP} 4 \\
\mathrm{CA} 1\end{array}$} & $\mathbf{0}$ & 0,5 & 1 & 1,5 & 2 \\
\hline & \multicolumn{5}{|c|}{$\begin{array}{l}\text { Consulta la bibliografía recomendada; selecciona ideas } \\
\text { relevantes e interesantes; cita datos y declaraciones de expertos, } \\
\text { procedentes de fuentes fiables; contextualiza históricamente la } \\
\text { noticia, buscando información sobre el tema, los personajes y el } \\
\text { acontecimiento. }\end{array}$} \\
\hline \multirow[b]{2}{*}{$\begin{array}{l}\text { Analiza el } \\
\text { periódico } \\
\mathrm{CP} 2, \mathrm{CP} 5, \mathrm{CP} 6 \\
\mathrm{CA} 2\end{array}$} & $\mathbf{0}$ & 0,5 & 1 & 1,5 & 2 \\
\hline & \multicolumn{5}{|c|}{$\begin{array}{l}\text { Responde en profundidad a varios de los problemas; maneja } \\
\text { información fiable y experta, procedente de la bibliografía } \\
\text { recomendada; pone ejemplos concretos, extraídos del periódico } \\
\text { elegido; reflexiona de forma crítica y razonada sobre el } \\
\text { periodismo actual. }\end{array}$} \\
\hline \multirow{2}{*}{$\begin{array}{l}\text { Redacta el } \\
\text { reportaje } \\
\text { CC5 } \\
\text { CP2, CP3, CP7 } \\
\text { CA5 }\end{array}$} & $\mathbf{0}$ & 1 & 2 & 3 & 4 \\
\hline & \multicolumn{5}{|c|}{$\begin{array}{l}\text { Cuida la expresión escrita y la exposición de las ideas; se ajusta } \\
\text { a la extensión indicada; contiene, al menos, un título, una } \\
\text { entradilla, dos destacados y un despiece; sigue las pautas dadas } \\
\text { en clase para titular, redactar y estructurar el reportaje. }\end{array}$} \\
\hline
\end{tabular}

Jornadas de Formación e Innovación Docente del Profesorado | № 2 (2019) Esta obra se distribuye con la licencia Creative Commons Reconocimiento-NoComercial-SinObraDerivada Internacional (CC BY-NC-ND 4.0.) 


\begin{tabular}{|l|c|c|c|c|c|}
\hline $\begin{array}{l}\text { Diseña el } \\
\text { reportaje } \\
\text { CC5 } \\
\text { CP2, CP7 / CA5 }\end{array}$ & $\mathbf{0}$ & $\mathbf{0 , 5}$ & $\mathbf{1}$ & $\mathbf{1 , 5}$ & $\mathbf{2}$ \\
\cline { 2 - 6 } & \multicolumn{5}{|c|}{$\begin{array}{l}\text { Cuida la presentación formal y el estilo; destaca los principales } \\
\text { elementos de redacción; inserta adecuadamente las imágenes. }\end{array}$} \\
\hline $\begin{array}{l}\text { Difunde el } \\
\text { reportaje } \\
\text { CP3, CP8 } \\
\text { CA5, CA6 }\end{array}$ & $\mathbf{0}$ & $\mathbf{0 , 2 5}$ & $\mathbf{0 , 5}$ & $\mathbf{0 , 7 5}$ & $\mathbf{1}$ \\
\cline { 2 - 5 } & \multicolumn{3}{|l|}{$\begin{array}{l}\text { Actividad voluntaria que permite sumar hasta 1 punto extra. Se } \\
\text { ajusta al tiempo; cuida la comunicación oral y visual; aborda } \\
\text { todos los contenidos: periódico, análisis y reportaje. }\end{array}$} \\
\hline
\end{tabular}

\section{Aplicación del CIMA}

\section{Relato resumido de las sesiones}

El relato resumido de las sesiones se nutre fundamentalmente de mi diario del profesor, una herramienta que me ha permitido tomar notas de campo de los momentos más interesantes y significativos que se han producido durante las clases. De esta forma he podido registrar cómo estaba funcionando realmente el ciclo: el clima de trabajo, mi actuación como docente, el desarrollo de las actividades o las dificultades encontradas.

\section{Sesión 1: martes, 8 de octubre de 2019.}

Una semana antes de comenzar el CIMA, los estudiantes respondieron al cuestionario inicial de preguntas cerradas (A1). Esto me permitió saber cuáles eran sus modelos mentales de partida, esto es, sus conocimientos previos acerca de los contenidos conceptuales que iban a trabajar en el ciclo. El tiempo programado (10 minutos) resultó insuficiente. La mayoría de los estudiantes respondió a la prueba en el aula, a través de la plataforma de enseñanza virtual, pero hubo algunos que la hicieron en los días siguientes, en horario no lectivo, porque tuvieron problemas técnicos o porque no asistieron a clase.

La primera sesión del CIMA consistió en una clase teórica sobre el tema 1, titulado «El nacimiento de la prensa 
en los primeros tiempos de la imprenta» (A2). La duración de la actividad fue de 120 minutos, un tiempo que he distribuido de la misma manera en todas las clases teóricas: 1) empezamos recordando los principales problemas abordados en la clase anterior y aclaramos dudas (10 minutos); 2) expongo el tema, desarrollando los contenidos conceptuales esenciales a partir de preguntas, y acabamos esbozando unas conclusiones mínimas (60 minutos); 3) realizamos estudios de caso a partir del comentario y la discusión de periódicos, testimonios, ilustraciones, mapas, vídeos (40 minutos); 4) recomiendo lecturas de profundización, novelas, películas, series, recursos web, etc. (10 minutos).

\section{Sesión 2: miércoles, 9 de octubre de 2019.}

En la segunda sesión del ciclo, nos trasladamos al aula de informática para tener la primera clase práctica. Los estudiantes ocuparon el nuevo espacio de aprendizaje simulando redacciones periodísticas a pequeña escala, en función de los equipos de trabajo que habían formado en las semanas previas. Esta ocupación fue especialmente complicada en el caso del grupo 1 debido al elevado número de estudiantes: 85 personas para 35 ordenadores. Hubo algunos equipos que se terminaron de constituir durante esta sesión.

En los primeros 10 minutos, los estudiantes respondieron individualmente al cuestionario inicial de autoevaluación (A3) a través de la plataforma de enseñanza virtual. La encuesta consta de 12 preguntas - diez cerradas y dos abiertas- y les permite reflexionar sobre la progresión de su propio aprendizaje. Mediante las preguntas cerradas pueden calificar -en una escala que va del excelente al insuficiente- su nivel o su capacidad en relación con los contenidos procedimentales y actitudinales del ciclo. En las preguntas abiertas, que no se responden en la autoevaluación inicial, pueden expresarse libremente sobre cómo perciben lo que han aprendido: qué les ha gustado y qué se podría mejorar, cómo ha sido su experiencia 
de aprendizaje colaborativo o cuáles son las dificultades personales que han encontrado.

Tras la autoevaluación, dedicamos 30 minutos a realizar una breve pero necesaria introducción sobre el género periodístico del reportaje (A4), con el objetivo de proporcionar a los estudiantes unos conocimientos teóricos básicos, ya que no habían cursado previamente ninguna asignatura de redacción.

Durante la presentación de la práctica (A5), a la que dedicamos 20 minutos, expliqué en qué consiste el modelo metodológico de periodismo-acción en el aula y mostré la guía que podían consultar en la plataforma de enseñanza virtual. Durante la segunda parte de la clase, los estudiantes eligieron el periódico (A6), sobre el que querían realizar la práctica, de una preselección que contenía 15 opciones. Al final de la actividad, en la que los estudiantes invirtieron cerca de una hora, no sólo habían elegido un periódico y se habían descargado la copia digital, sino que también habían empezado a leer la portada y la noticia principal.

\section{Sesión 3: martes, 15 de octubre de 2019.}

Esta sesión consistió en una clase teórica sobre el tema 2, titulado «Fijación y evolución del gaceterismo en el Estado absolutista moderno» (A7).

\section{Sesión 4: miércoles, 16 de octubre de 2019.}

Esta sesión consistió en una clase práctica sobre cómo buscar y seleccionar fuentes de información fiables. Durante la primera hora, los estudiantes empezaron a consultar la bibliografia recomendada (A8), con el objetivo de seleccionar aquellas ideas que considerasen relevantes, interesantes e incluso curiosas, como si hubieran obtenido esos testimonios después de entrevistar a expertos en la materia. A continuación, en la segunda hora de la clase, los estudiantes pasaron a contextualizar históricamente la noticia principal de su periódico (A9), para lo que debían buscar información sobre el acontecimiento histórico y los 
principales personajes involucrados. Cuando finalizó la sesión, les advertí de que debían proseguir estas actividades en horario no presencial.

\section{Sesión 5: martes, 22 de octubre de 2019.}

Esta sesión consistió en una clase teórica sobre el tema 3, titulado «Las batallas del periodismo británico» (A10).

\section{Sesión 6: miércoles, 23 de octubre de 2019.}

En esta clase práctica, los estudiantes destinaron los primeros 60 minutos a analizar el periódico elegido (A11). En el análisis, debían abordar todos los problemas de investigación planteados en el mapa de contenidos, si bien podían alcanzar diferentes niveles de profundidad en cada uno de ellos. En la segunda parte de la sesión, expliqué brevemente cómo elaborar un reportaje periodístico de interés histórico (A12). A continuación, cada redacción empezó a trabajar en la redacción y en el diseño de sus reportajes. Al final de la sesión, les hice saber a todos que tanto el análisis como la elaboración del reportaje eran actividades que había que continuar en horario no presencial. Les comenté la pertinencia de empezar a preparar un borrador y les insté a cuidar la expresión escrita, el estilo y la presentación formal. Por último, pregunté cuántas redacciones estaban sopesando la idea de presentar públicamente su reportaje en la última sesión del ciclo; aproveché para explicarles brevemente en qué iba a consistir esa actividad y qué esperaba de sus intervenciones.

\section{Sesión 7: martes, 29 de octubre de 2019.}

Esta sesión consistió en la última clase teórica. Abordamos el tema 4, titulado «La prensa política al servicio de la revolución» (A13). Tras hacer balance de este tipo de actividad, observo que la asistencia - no era obligatoriaha ido decreciendo, sobre todo en los grupos 3 y 4 , y que la participación de los estudiantes en el aula, tanto en los estudios de caso como en los debates, ha sido desigual: entre grupos, la interacción lograda en el grupo 1 ha sido 
superior a la de los grupos 3 y 4 en términos de intensidad y de razonamiento crítico; dentro de cada grupo, siempre intervienen los mismos estudiantes, en torno a un $20 \%$ del total.

\section{Sesión 8: miércoles, 30 de octubre de 2019.}

Dedicamos la última sesión del ciclo a la difusión del reportaje y a la discusión de los resultados (A14), una clase práctica en la que los estudiantes presentaron sus creaciones en el aula. Las confrontaron con las de sus compañeros y se sometieron a la coevaluación, que se realizó en forma de comentarios y mediante un sistema de tarjetas de colores. Pese a tratarse de una actividad de carácter voluntario, el 65\% de las redacciones -15 de 23- presentó públicamente sus reportajes. Como profesor, me limité a moderar la evaluación entre iguales y a conectar las valoraciones de los estudiantes con los problemas y contenidos.

Esta actividad superó mis mejores expectativas en cuanto a la implicación de los estudiantes y a la calidad de sus intervenciones, de manera que el tiempo previsto (100 minutos) resultó insuficiente. En consecuencia, no dio tiempo a realizar las otras dos actividades programadas. La solución, consensuada con los estudiantes, consistió en que respondieran al cuestionario final de autoevaluación (A15) y al cuestionario final de preguntas cerradas (A16) fuera del aula, a través de la plataforma, durante las 48 horas siguientes.

También en horario no presencial, los estudiantes debían revisar sus reportajes, teniendo en cuenta los comentarios que habían recibido de los compañeros en la última sesión. Previa petición del alumnado, el plazo para entregar la versión corregida y acabada del reportaje (A17), a través de la plataforma de enseñanza virtual, se amplió del 1 al 4 de noviembre. 


\section{Evaluación del aprendizaje de los estudiantes}

He evaluado el aprendizaje de los estudiantes mediante dos instrumentos: el cuestionario individual de elección múltiple y la rúbrica para calificar la práctica grupal del reportaje. La decisión de diseñar un cuestionario de preguntas cerradas obedece a dos motivos. El primero se refiere al elevado número de estudiantes que han participado en el ciclo, cerca de 200, lo que podía dificultar el análisis de los resultados proporcionados por un cuestionario de preguntas abiertas. El segundo motivo es que la evaluación -sobre todo, de los contenidos procedimentales y actitudinales- también se ha realizado de forma cualitativa, mediante la rúbrica del reportaje. Conforme al modelo metodológico de periodismo-acción en el aula, los estudiantes han tenido que responder a preguntas abiertas, indirectas y estimulantes, así como interpretar fenómenos y simular situaciones profesionales de interés.

Sin embargo, sólo en el caso del cuestionario he podido comparar los conocimientos iniciales y finales de los estudiantes, así como representar gráficamente la evolución de sus modelos mentales mediante escaleras de aprendizaje. En el caso de la rúbrica, esto no ha sido posible por una simple cuestión de tiempo: el segundo reportaje se entrega a comienzos de diciembre y el tercero, ya en enero, mientras que la versión definitiva de este artículo debía aprobarse antes del 26 de noviembre. 
ESCALERA INICIAL

Cuestionarios: 181

Valor máximo: 5

Promedio: 1,5

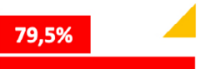

Nivel 1

0-2 preguntas

correctas

\section{$28 \%$}

Nivel 1

$0-2$ preguntas

correctas

No asiste a clase

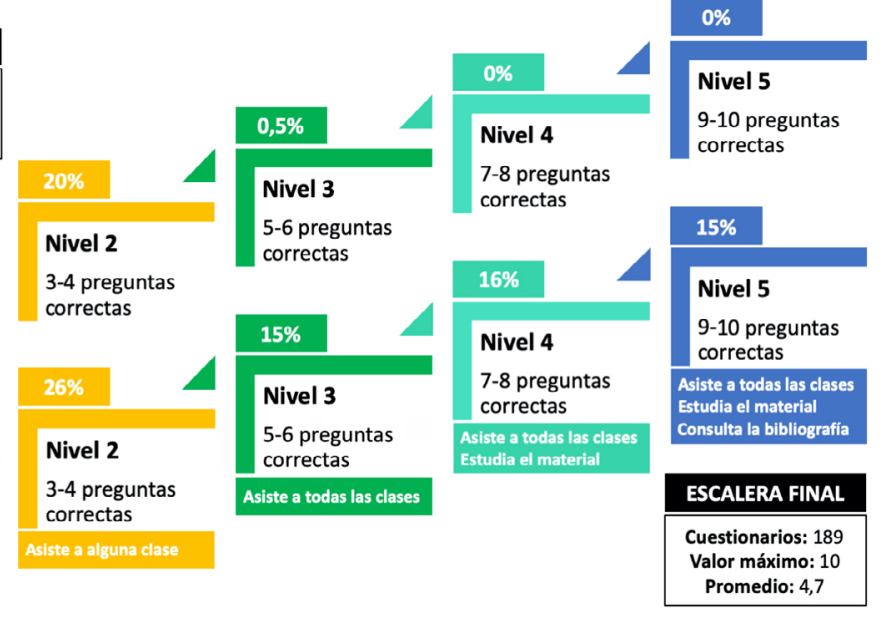

Promedio: 4,7

Figura 3. Escaleras de aprendizaje basadas en el cuestionario de elección múltiple.

En lo que se refiere al cuestionario de elección múltiple, tal y como puede verse en la Figura 3, he clasificado las respuestas a las preguntas en grupos que reflejan un patrón común -el número de preguntas respondidas correctamente- y los he ordenado en escaleras, colocando al lado de cada grupo (o escalón) el porcentaje de estudiantes que están en ese nivel. Sólo en la escalera final, he identificado los obstáculos de aprendizaje entre un nivel y el siguiente y los he formulado con una frase.

Lo primero que se desprende de la escalera inicial es el bajo nivel de conocimientos que, antes de comenzar el ciclo, los estudiantes tenían sobre el origen del periodismo. Me sorprende que el $80 \%$ de los estudiantes no fuera capaz de responder correctamente a más de dos preguntas, puesto que todos habían cursado previamente Historia Social de la Comunicación, una asignatura «llave» en la que, en principio, se abordan algunos de los contenidos conceptuales que se preguntan en el cuestionario.

Jornadas de Formación e Innovación Docente del Profesorado | № 2 (2019) Esta obra se distribuye con la licencia Creative Commons Reconocimiento-NoComercial-SinObraDerivada 
La escalera final, en cambio, denota una progresión eso sí, moderada- en el aprendizaje de los estudiantes a lo largo del ciclo. El análisis de los resultados obtenidos en el cuestionario final revela que el $46 \%$ del alumnado ya sí responde correctamente, al menos, a la mitad de las preguntas y, por tanto, obtiene una calificación igual o superior a 5 en la prueba; incluso el 15\% es capaz de llegar al sobresaliente. Sin embargo, no me deja satisfecho el elevado porcentaje de estudiantes que no ha superado la prueba: más de la mitad. Esta circunstancia se debe, en mi opinión, a dos factores. El primero tendría que ver con el nivel de complejidad del cuestionario, constituido por preguntas de elección múltiple, que suelen generar duda e inseguridad entre algunos estudiantes. El segundo factor, que explica el alto número de suspensos, estaría relacionado con la inasistencia a clase y con un insuficiente trabajo autónomo por parte del alumnado. He podido hablar de manera informal con algunos de los estudiantes que se encuentran en este nivel y me han reconocido que, antes de responder la prueba final, no estudiaron el material disponible en la plataforma de enseñanza virtual ni consultaron la bibliografia recomendada.

En cualquier caso, las escaleras derivadas del cuestionario me han servido para revisar este instrumento de evaluación, de manera que, en las dos pruebas siguientes, modificaré el tipo de pregunta y, de las tres opciones, sólo una respuesta será correcta. Además, tomando como referencia los obstáculos detectados, recompensaré la asistencia a las clases teóricas con un punto extra en la calificación de los cuestionarios e insistiré en la necesidad de estudiar el material y de consultar la bibliografia.

En lo que se refiere a la práctica, ya he reconocido que no he podido comparar los conocimientos iniciales y finales de los estudiantes. Sin embargo, he decidido -como solución provisional- cotejar los resultados de la rúbrica, obtenidos tras la evaluación del primer reportaje, con las 
calificaciones proporcionadas por los mismos estudiantes en el cuestionario final de autoevaluación. Tras analizar las escaleras de aprendizaje, representadas en la Figura 4, puede observarse que, en los niveles $2-4$, se ha producido un alto grado de coincidencia entre lo que sienten los estudiantes que han aprendido -acerca del análisis, la redacción o el diseño- y la calificación que, finalmente, han obtenido tras la evaluación del profesor. No obstante, hay dos niveles -el 1 y el 5 - en los que se ha dado un desequilibro pronunciado entre la autoevaluación y la rúbrica: a juicio del docente, los estudiantes no han buscado y seleccionado información, ni han difundido su reportaje en el aula, tan bien como ellos pensaban.

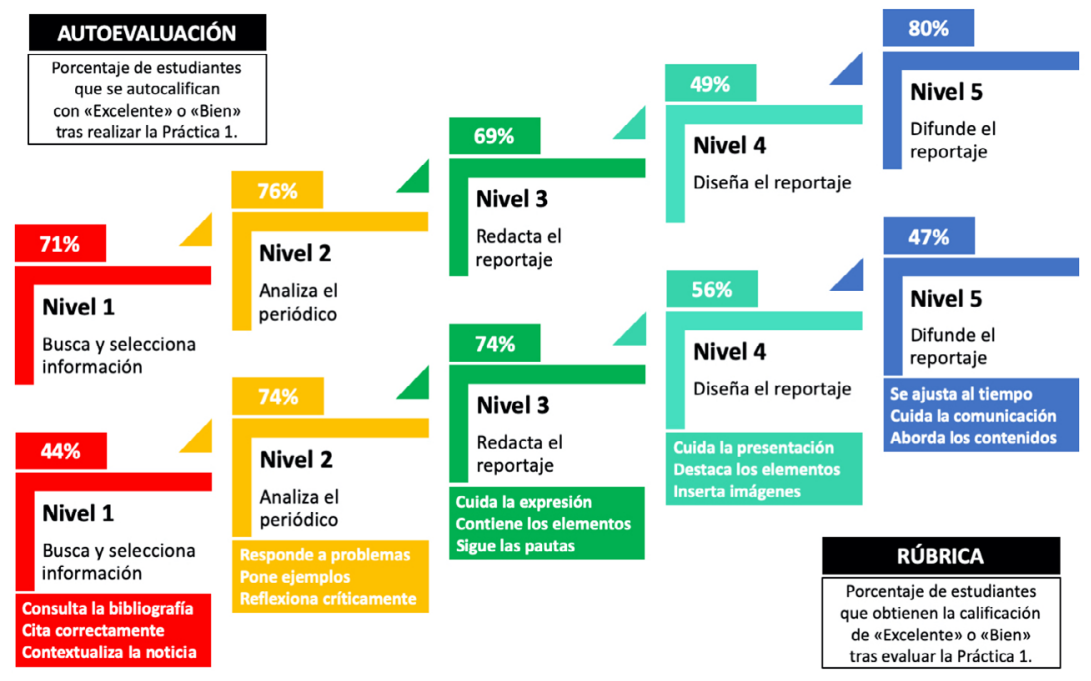

Figura 4. Escaleras de aprendizaje basadas en la autoevaluación final y en la rúbrica de la práctica.

\section{Evaluación del CIMA}

Para evaluar mi actuación docente y la experimentación llevada a cabo mediante el CIMA, he utilizado fundamentalmente dos instrumentos: el diario del profesor y una encuesta de opinión a los estudiantes. Ambos me han aportado información valiosa y me han facilitado la reflexión y la toma de decisiones. El primer instrumento, el 
diario del profesor, me ha permitido evaluar -y rectificar sobre la marcha- tanto el funcionamiento del ciclo como mi propia intervención (Rivero y Porlán, 2017). A través del segundo instrumento, la encuesta, los estudiantes han podido evaluar la experiencia de aprendizaje que les ha proporcionado el CIMA. Esta herramienta me ha servido para identificar fortalezas y debilidades del proceso de experimentación. Se trata de un cuestionario anónimo, voluntario y no evaluable, que 162 estudiantes respondieron, una vez finalizado el ciclo, a través de la plataforma de enseñanza virtual. La encuesta contiene diez ítems de índole cuantitativa, que permiten al alumnado mostrar su grado de acuerdo mediante una escala de tipo Likert.

Tal y como puede observarse en la Tabla 3, el grado de conformidad que el alumnado ha expresado en todas las preguntas de la encuesta es, por lo general, bastante alto. Destaca, por ejemplo, cómo ocho de cada diez encuestados valoran positivamente la planificación, organización y orientación de las actividades, el papel autónomo y activo que se les ha dado o la motivación que han sentido para interesarse más por la asignatura. Por el contrario, hay varios ítems que permiten un amplio margen de mejora, como los relativos al aula, a las tareas, a las preguntas o al pensamiento crítico.

Tabla 3. Porcentaje de estudiantes que están totalmente de acuerdo o de acuerdo con las afirmaciones de la encuesta de opinión.

\begin{tabular}{|c|l|c|}
\hline Pregunta & \multicolumn{1}{|c|}{ Enunciado } & Final \\
\hline 1 & $\begin{array}{l}\text { Las actividades que hemos realizado estaban bien } \\
\text { planificadas y organizadas. }\end{array}$ & $80 \%$ \\
\hline 2 & $\begin{array}{l}\text { Hemos recibido las orientaciones adecuadas para realizar } \\
\text { las actividades. }\end{array}$ & $83 \%$ \\
\hline 3 & $\begin{array}{l}\text { Hemos tenido un papel autónomo y activo en el desarrollo } \\
\text { de las actividades. }\end{array}$ & $82 \%$ \\
\hline
\end{tabular}

Jornadas de Formación e Innovación Docente del Profesorado | № 2 (2019) Esta obra se distribuye con la licencia Creative Commons Reconocimiento-NoComercial-SinObraDerivada Internacional (CC BY-NC-ND 4.0.) 


\begin{tabular}{|c|l|c|}
\hline 4 & $\begin{array}{l}\text { Las preguntas que se nos han planteado han sido } \\
\text { interesantes y relevantes. }\end{array}$ & $66 \%$ \\
\hline 5 & Las tareas que hemos realizado han sido estimulantes. & $64 \%$ \\
\hline 6 & $\begin{array}{l}\text { Se ha generado un entorno en el que hemos aprendido a } \\
\text { pensar críticamente. }\end{array}$ & $61 \%$ \\
\hline 7 & $\begin{array}{l}\text { Se ha promovido un entorno en el que hemos aprendido a } \\
\text { trabajar en equipo. }\end{array}$ & $73 \%$ \\
\hline 9 & $\begin{array}{l}\text { El aula de informática reúne las condiciones adecuadas } \\
\text { para trabajar en equipo. }\end{array}$ & $55 \%$ \\
\hline 10 & $\begin{array}{l}\text { Se nos ha motivado para que nos interesemos más por la } \\
\text { asignatura. }\end{array}$ \\
\hline
\end{tabular}

Finalizado el ciclo, una de las principales incorporaciones que voy a hacer a mi práctica docente habitual es la que se refiere a la propuesta metodológica que yo mismo he bautizado con el nombre de periodismo-acción en el aula. Por lo que he podido hablar con los estudiantes en las clases y por lo que han expresado tanto en la encuesta de opinión como en la autoevaluación, la experiencia de aprendizaje ha sido positiva y enriquecedora. Sirva este testimonio anónimo, expresado en la autoevaluación, como muestra: "Aunque también ha sido una forma más autónoma y divertida de aprender, lo que más me ha gustado, sin duda, ha sido la sensación de trabajar en una redacción, la oportunidad de ponerme en la piel de un periodista y empezar a conocer mi futura profesión». Para mí ha sido un reto enseñar historia del periodismo desde el periodismo, lo que me ha llevado a cambiar mis planteamientos docentes y a proponer problemas y procesos profesionales, de manera que los estudiantes han terminado implicándose en su aprendizaje -desde el cuestionamiento, la búsqueda y la interpretación de información- para crear nuevos conocimientos. 
La actividad destinada a la difusión de los reportajes en el aula, en la que hemos implementado la evaluación entre iguales, ha sido uno de los momentos más enriquecedores de todo el ciclo. Tengo la impresión de que el aprendizaje ha sido más auténtico y estimulante para la mayoría de los estudiantes. Sirva como ejemplo este testimonio: «Me ha gustado ser evaluada por el resto de la clase, ha sido una forma muy útil de corregir errores». La sesión ha servido para fomentar su capacidad analítica e interpretativa, de manera que han puesto en juego los conceptos y las destrezas aprendidos a la vez que han confrontado ideas, sopesándolas, aceptándolas o rechazándolas. Me ha sorprendido gratamente el alto nivel de muchas intervenciones, basadas en sólidos argumentos y en razonamientos críticos.

Otro aspecto de la experiencia que pretendo incorporar a mi práctica docente habitual es el cuestionario inicial-final de preguntas cerradas, aunque con algunas modificaciones: en aras de reducir su supuesta complejidad, cada pregunta seguirá teniendo varias respuestas, pero sólo una opción será correcta; además, para evitar un posible fraude, los estudiantes siempre realizarán la prueba en el aula y en horario de clase.

Una de las principales dificultades que ha lastrado el desarrollo del ciclo ha sido el espacio de aprendizaje, descrito en el primer apartado, y la masificación de los grupos de clase. Por ejemplo, la actividad destinada a la difusión y coevaluación de los reportajes se desarrolló en el aula de informática, donde no es posible crear un espacio circular en torno al que distribuir a los estudiantes para que discutan y conversen de manera natural. Sin embargo, a diferencia del ciclo anterior, esta vez sí me he atrevido a experimentar el ciclo en los tres grupos en los que impartía la asignatura. En mi opinión, la decisión de formar equipos de trabajo de entre ocho y diez integrantes ha sido un acierto, puesto que los 199 estudiantes que han 
participado en el CIMA se han agrupado en 23 redacciones. De esta forma, he tenido tiempo suficiente para orientar y ayudar a todos los grupos en las clases prácticas. Además, he sido capaz de evaluar sus reportajes en un plazo inferior a diez días.

Fruto de esta evaluación reflexiva del proceso, he seleccionado aquellos principios didácticos que considero más valiosos y que, por tanto, han guiado la experimentación durante el ciclo, conforme a un modelo alternativo «centrado en el alumno y el aprendizaje» (García Pérez y Porlán, 2017).

- Cederé cada vez más el control a mis estudiantes, reestructurando la configuración física y emocional del aula, para que estos asuman un papel activo y participativo en el proceso de aprendizaje (Finkel, 2008).

- En relación con los contenidos, asumiré una concepción integradora que contemple conceptos, procedimientos y actitudes (García Díaz, Porlán y Navarro, 2017) e intentaré conectarlos con los problemas de la disciplina y de la profesión, así como convertir el conocimiento en un proceso, de manera que sean mis estudiantes, mediante problemas enfocados, quienes lo construyan y quienes descubran por sí mismos las conclusiones (Finkel, 2008).

- Comenzaré siempre con mis estudiantes, en lugar de con lo que dicta la disciplina o interesa al profesor (Bain, 2007).

- En relación con la metodología, procuraré crear, en mis clases, experiencias de aprendizaje basadas en el equilibrio entre lo sistemático y lo desordenado, entre lo cotidiano y lo innovador (Bain, 2007) y buscaré acercarme cada vez más a un modelo basado en la reelaboración de las ideas de los estudiantes (De Alba y Porlán, 2017). 
- Dejaré que hablen mis estudiantes, en el sentido de que piensen y aprendan a participar durante las discusiones en clase (Finkel, 2008).

- En mis asignaturas diseñaré actividades basadas en el aprendizaje colaborativo (Oakley et al., 2004).

- En relación con la evaluación, plantearé un modelo que analice y compare los modelos iniciales y finales de mis estudiantes, que ponga el acento sobre su aprendizaje, y no tanto en el rendimiento, que incorpore la autoevaluación y la coevaluación en mis clases o que tenga en cuenta la influencia de la evaluación en la motivación y la autoestima de mi alumnado (Bain, 2007; Rivero y Porlán, 2017). 


\section{Referencias bibliográficas}

Bain, K. (2007). Lo que hacen los mejores profesores universitarios. Valencia: Universitat de València.

De Alba, N. y Porlán, R. (2017). La metodología de enseñanza. En Porlán, R. (Coord.) Enseñanza universitaria. Cómo mejorarla (pp. 37-53). Madrid: Ediciones Morata.

Finkel, D. (2008). Dar clase con la boca cerrada. Valencia: Universitat de València.

García Díaz, E., Porlán, R. y Navarro, E. (2017). Los fines y los contenidos de enseñanza. En Porlán, R. (Coord.) Enseñanza universitaria. Cómo mejorarla (pp. 55-72). Madrid: Ediciones Morata.

García Pérez, F. F. (2000). Un modelo didáctico alternativo para transformar la educación: el Modelo de Investigación en la Escuela. Scripta Nova. Revista Electrónica de Geografia y Ciencias Sociales, 64. Recuperado de http:/ / www.ub.edu/geocrit/sn-64.htm

García Pérez, F. F. y Porlán, R. (2017). Los Principios Didácticos y el Modelo Didáctico Personal. En Porlán, R. (Coord.) Enseñanza universitaria. Cómo mejorarla (pp. 93-104). Madrid: Ediciones Morata.

Oakley, B.; Felder, R. M.; Brent, R. y Elhajj, I. (2004). Turning Student Groups into Effective Teams. Journal of Student Centered Learning, 2(1), 9-34.

Piqué, B. y A. Forés (2012). Propuestas metodológicas para la educación superior. Barcelona: Universitat de Barcelona. Recuperado de http:/ / hdl.handle.net/2445/3070

Porlán, R. (Coord.). (2017). Enseñanza universitaria. Cómo mejorarla. Madrid: Ediciones Morata.

Rivero, A. y Porlán, R. (2017). La evaluación en la enseñanza universitaria. En Porlán, R. (Coord.) Enseñanza universitaria. Cómo mejorarla (pp. 74-91). Madrid: Ediciones Morata. 